\title{
Frequent aberrant DNA methylation of $A B C B 1$, FOXC1, PPP2R2B and PTEN in ductal carcinoma in situ and early invasive breast cancer
}

\author{
Aslaug Aa Muggerud $+1,2$, Jo Anders Rønneberg $+1,2$, Fredrik Wärnberg33, Johan Botling4, Florence Busato9, \\ Jovana Jovanovic ${ }^{5}$, Hiroko Solvang 1,10, Ida Bukholm6, Anne-Lise Børresen-Dale1,2, Vessela N Kristensen*1,5,7, \\ Therese Sørlie1,8 and Jörg Tost 9
}

\begin{abstract}
Introduction: Ductal carcinoma in situ (DCIS) is a non-invasive lesion of the breast that is frequently detected by mammography and subsequently removed by surgery. However, it is estimated that about half of the detected lesions would never have progressed into invasive cancer. Identifying DCIS and invasive cancer specific epigenetic lesions and understanding how these epigenetic changes are involved in triggering tumour progression is important for a better understanding of which lesions are at risk of becoming invasive.

Methods: Quantitative DNA methylation analysis of ABCB1, CDKN2A/P16INK4a, ESR1, FOXC1, GSTP1, IGF2, MGMT, MLH1, PPP2R2B, PTEN and RASSF1A was performed by pyrosequencing in a series of 27 pure DCIS, 28 small invasive ductal carcinomas (IDCS), 34 IDCs with a DCIS component and 5 normal breast tissue samples. FOXC1, ABCB1, PPP2R2B and PTEN were analyzed in 23 additional normal breast tissue samples. Real-Time PCR expression analysis was performed for FOXC1.

Results: Aberrant DNA methylation was observed in all three diagnosis groups for the following genes: $A B C B 1, F O X C 1$, GSTP1, MGMT, MLH1, PPP2R2B, PTEN and RASSF1A. For most of these genes, methylation was already present at the DCIS level with the same frequency as within IDCs. For FOXC1 significant differences in methylation levels were observed between normal breast tissue and invasive tumours $(P<0.001)$. The average DNA methylation levels were significantly higher in the pure IDCs and IDCs with DCIS compared to pure DCIS ( $P=0.007$ and $P=0.001$, respectively). Real-time PCR analysis of FOXC1 expression from 25 DCIS, 23 IDCs and 28 normal tissue samples showed lower gene expression levels of FOXC1 in both methylated and unmethylated tumours compared to normal tissue $(P<0.001)$. DNA methylation levels of FOXC1, GSTP1, ABCB1 and RASSF1A were higher in oestrogen receptor (ER) positive vs. ER negative tumours; whereas methylation levels of FOXC1, $A B C B 1, P P P 2 R 2 B$ and PTEN were lower in tumours with a TP53 mutation.

Conclusions: Quantitative methylation analysis identified $A B C B 1, F O X C 1, P P P 2 R 2 B$ and PTEN as novel genes to be methylated in DCIS. In particular, FOXC1 showed a significant increase in the methylation frequency in invasive tumours. Low FOXCI gene expression in both methylated and unmethylated DCIS and IDCs indicates that the loss of its expression is an early event during breast cancer progression.
\end{abstract}

\section{Introduction}

The multistep model of breast cancer progression suggests a transition from normal epithelium to invasive carcinoma via intraductal hyperplasia and in situ carcinoma

* Correspondence: vessela.kristensen@medisin.uio.no

+ Contributed equally

1 Department of Genetics, Institute for Cancer Research, Oslo University

Hospital Radiumhospitalet, Montebello, Oslo, N-0310, Norway
[1]. These presumptive precursor lesions are currently defined by their histological features. Ductal carcinoma in situ (DCIS) is a pre-invasive lesion with diverse histological morphologies and molecular alterations [2]. The risk of DCIS progressing to invasive carcinoma is not well ascertained and robust biomarkers capable of stratifying the most aggressive from the more benign forms of the disease are currently lacking. 
Cancer progression is due to the accumulation of genomic alterations leading to oncogene overexpression and tumour suppressor loss inducing growth advantage and clonal expansion. The transition of DCIS to invasive ductal cancer (IDC) is a poorly understood key event in breast tumour progression. Copy number alterations are already present in DCIS but their frequency tends to increase in IDCs [3]. Such genomic aberrations lead to altered gene expression, and comprehensive gene expression studies comparing DCIS and IDCs have identified stage-specific markers ([4-6] and Muggerud et al., submitted) along with a gene expression classifier which differed between DCIS and invasive breast cancer [7]. On the other hand, the frequency of TP53 mutations in DCIS is similar to what is observed in invasive tumours and in situ and invasive components from the same tumour exhibit the same mutations, indicating the same cellular origin of the two components [8-10].

Epigenetic changes are considered to be an early event during tumour development and one of the hallmarks of cancer [11]. Hypermethylation of CpG islands represents an alternative mechanism to inactivate tumour suppressor genes and is a prevalent early molecular marker for cancer. Specific patterns of $\mathrm{CpG}$ island methylation could result from clonal selection of cells having growth advantages due to silencing of associated tumour suppressor genes, DNA repair genes, cell-cycle regulators and transcription factors. Previous candidate gene studies investigated promoter hypermethylation of in situ lesions and identified aberrant methylation at the promoters of GSTP1, CyclinD2, RARB2, Twist, RASSF1A, HIN-1, $C D K N 2 A, 14-3-3 \sigma$ and $A P C 1$ [12-17]. However, only GSTP1 promoter hypermethylation was reported to progress in frequency during breast carcinogenesis [12].

Identification of early epigenetic changes in DCIS lesions might give valuable markers for early detection and may contribute to the understanding of how these changes affect the progression of the disease. The aim of this study was to identify informative progression markers by methylation analyses of eleven genes known to be methylated in breast tumours or breast cancer cell lines; ABCB1 [18], CDKN2A/p16INK4a [19], ESR1 [20], GSTP1

Table 1: Clinicopathological factors

\begin{tabular}{|c|c|c|c|}
\hline & No. DCIS & No. Invasive & No. Mixed \\
\hline Diagnosis & 27 & 28 & 34 \\
\hline \multicolumn{4}{|l|}{ Oestrogen receptor status } \\
\hline Positive & 19 & 23 & 24 \\
\hline Negative & 8 & 4 & 8 \\
\hline \multicolumn{4}{|l|}{$\begin{array}{l}\text { Progesterone receptor } \\
\text { status }\end{array}$} \\
\hline Positive & 20 & 21 & 20 \\
\hline Negative & 7 & 6 & 12 \\
\hline \multicolumn{4}{|l|}{ TP53 mutations } \\
\hline Wild type & 22 & 23 & 24 \\
\hline Mutant & 5 & 4 & 8 \\
\hline \multicolumn{4}{|l|}{ Ki67 } \\
\hline Positive & 6 & 6 & 14 \\
\hline Negative & 21 & 22 & 20 \\
\hline \multicolumn{4}{|l|}{ Grade, I to III and/or A to C } \\
\hline Grade A/I (DCIS/invasive) & 1 & 13 & $3 / 8$ \\
\hline Grade B/II (DCIS/invasive) & 13 & 10 & $8 / 17$ \\
\hline Grade C/III (DCIS/invasive) & 12 & 4 & $15 / 7$ \\
\hline
\end{tabular}


[21], IGF2 [22], MGMT [19], MLH1 [19], PPP2R2B [23], PTEN [24], RASSF1A [25] or displaying variation in breast cancer sub-type gene expression profiles; FOXC1.

In a series of 27 DCIS, 28 IDCs, 34 mixed cases (invasive tumours with in situ components) and 28 normal tissues we show that methylation of $\mathrm{CpG}$ islands is already detectable in DCIS with the same frequency as within IDCs.

\section{Materials and methods Patient material}

Patients with fresh frozen tumour samples, collected at the Fresh Tissue Biobank at the Department of Pathology, Uppsala University Hospital, Sweden, were selected from a population-based cohort of 854 women diagnosed between 1986 and 2004, with either one of three types of primary breast cancer lesions; a) pure DCIS, b) pure invasive breast cancer, $15 \mathrm{~mm}$ or less, or c) mixed lesions (invasive carcinoma with an in situ component). All histopathological specimens, both paraffin embedded (used in IHC analyses) and frozen (used in methylation and quantitative real-time polymerase chain reaction (qRT PCR) analyses), were re-evaluated by a breast pathologist. Seventy-seven percent of the pure DCIS samples have a DCIS component of $>70 \%$. Seventy-six percent of the invasive samples have a tumour content of $>70 \%$, while $79 \%$ of the mixed samples have a tumour/DCIS component of $>70 \%$. DCIS lesions were classified according to the European Organisation for Research and Treatment of Cancer (EORTC) classification system [26]. We denoted the grades $\mathrm{A}$ to $\mathrm{C}$ (corresponding to grades I to III) to make clear that in situ and invasive lesions were classified based on different systems. Invasive breast cancers were classified based on the Elston-Ellis classification system, grades I to III [27]. Twenty-eight samples of normal breast epithelium were collected at the Akershus University Hospital from women undergoing a biopsy for the suspicion of malignant disease but without any histological findings. Five of the normal samples had enough DNA to be used in the methylation analyses of all genes in all patients. Twenty-three additional normal tissues were included in the methylation analysis of FOXC1, $A B C B 1, P P P 2 R 2 B$ and PTEN and qRT-PCR analyses of $F O X C 1$. All patients signed a written consent to participate in the study, which has been approved by the regional ethical committee. Clinicopathological details of lesions are given in Table 1 . This study was designed to investigate differences in $\mathrm{CpG}$ methylation events between different diagnostic groups with a particular emphasis on identifying specific markers related to tumour progression from in situ to invasive cancer. We would like to emphasise that this study was not designed to study prognosis. For example, none of the DCIS patients died from breast cancer and only three experi- enced a local recurrence. Also, the patients were treated differently according to tumour characteristics. Hence, no follow-up data can be presented. The study was approved by the Ethics Committee at Uppsala University Hospital (Dnr 2005:118).

\section{Clinical endpoints}

The entire coding sequence of the TP53 gene (exon 2-11) was analyzed for mutations by sequencing using the 3730 DNA Analyzer (Applied Biosystems, Foster City, California, USA) [10]. Immunohistochemical staining (IHC) of paraffin embedded material was performed for the oestrogen receptor (ER), progesterone receptor (PR) and a proliferation marker (Ki-67). A cut-off limit for positive staining was chosen for ER $>10 \%$ (ER 6F11, Novocastra, Newcastle, UK), PR $>10 \%$ (PR 1A6, Novocastra) and Ki$67>10 \%$ (Ki-67 MIB-1, DAKO A/S, Glostrup, Denmark) stained tumour cells, irrespective of the intensity of the staining. Staining was performed in an automatic staining machine (Ventana Medical Systems, Tucson, AZ, USA).

\section{Pyrosequencing}

A total of $1 \mu \mathrm{g}$ of DNA was bisulphite converted using the MethylEasy $^{\text {Tum }}$ HT Kit for Centrifuge (Human Genetic Signatures, North Ryde, New South Wales, Australia) according to the manufacturer's instructions. Quantitative DNA methylation analysis of the bisulphite treated DNA was performed by pyrosequencing or, in case of several sequencing primers, by serial pyrosequencing [28]. Oligonucleotides for PCR amplification and pyrosequencing were synthesized by Biotez (Buch, Germany) and sequences are given in Additional file 1. Quantitative DNA methylation analysis was carried out on a PSQ 96 MD system with the PyroGold SQA Reagent Kit (Pyrosequencing, Biotage, Uppsala, Sweden) and results were analyzed using the Q-CpG software (V.1.0.9, Pyrosequencing $\mathrm{AB}$ ). Unmethylated commercial DNA (Qiagen, Valencia, CA, USA) and mixed human lymphocyte DNA (Promega, Madison, WI, USA) was analyzed in parallel to define the technical background. CpG-values for tumour and normal tissue samples are given in Additional file 2. Pyrograms for FOXC1 in six tumour samples and three normal tissue samples are given in Additional file 3.

\section{CDNA synthesis and real-time PCR analysis}

qRT-PCR was performed on 25 DCIS, 23 pure invasive carcinomas from the same cohort and 28 normal tissues. cDNA was synthesized in a total volume of $20 \mu \mathrm{l}$ with 100 ng total RNA using the High Capacity cDNA Reverse Transcription kit (Applied Biosystems,) and used as template for real-time PCR analysis with the TaqMan Gene Expression Assay for FOXC1 (Hs00559473_s1, Applied Biosystems,) on an ABI Prism 7900HT sequence detector system (Applied Biosystems). Universal human reference 


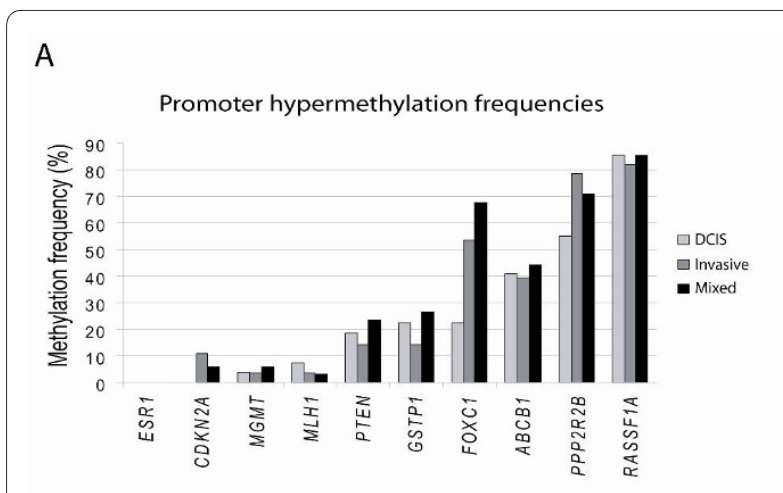

B

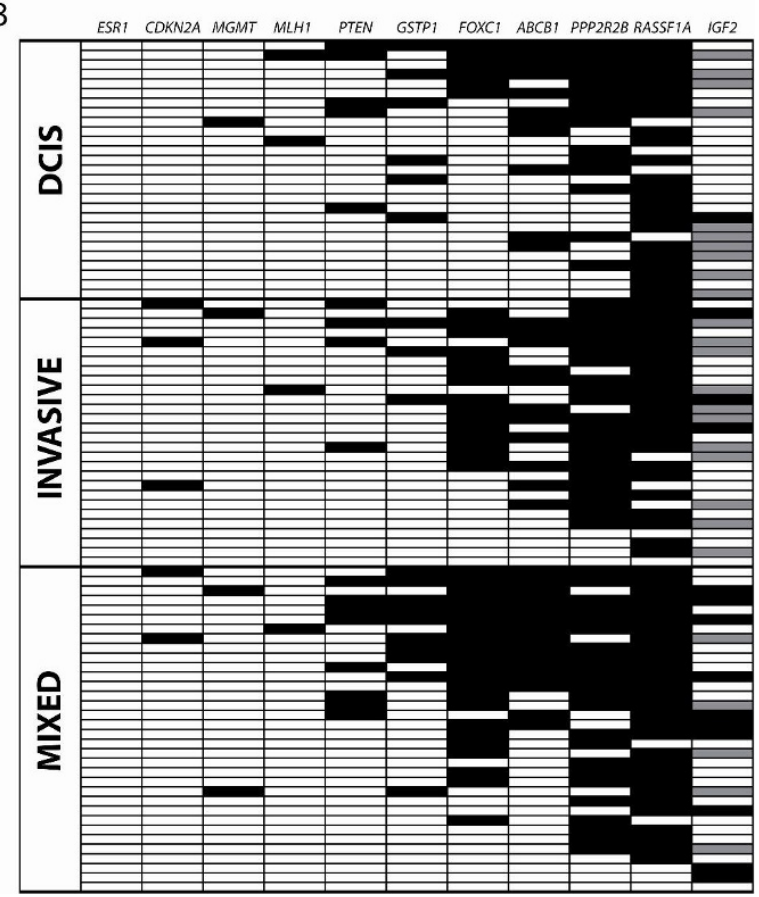

Figure 1 Methylation overview. A: Bar chart displaying promoter methylation frequencies across the three diagnosis groups. Methylation frequency is defined as the number of methylated samples within each category. The average values of methylation for all CpGs were calculated for each sample and each gene. A sample was scored as hypermethylated if the measured methylation values were two times above the standard deviation of the mean of the normal controls, and conversely, as hypomethylated if methylation values were below two times the standard deviation of the mean of the normal control tissues. DCIS $=$ light grey, pure invasive $=$ dark grey, and mixed $=$ black. $\mathbf{B}$ : Methylation overview per gene across the three diagnosis groups. Black boxes indicate methylated and white boxes indicate unmethylated samples. For the imprinted gene IGF2; white boxes indicate the expected allele-specific methylation, black boxes indicate hypermethylation, and grey boxes indicate hypomethylation.

RNA (Stratagene, La Jolla, CA, USA) was used to generate standard curves. Each sample was run in triplicate. Relative gene expression levels were determined using the standard curve method and normalized to the reference gene PGK1. PGK1 was selected as a reference gene because the expression of this gene was not statistically significant different across the patient groups $[29,30]$

\section{Statistics}

The average value of methylation for all CpGs in a target region was calculated for each sample and each gene. A sample was scored as aberrantly hypermethylated if the measured methylation values were two times above the standard deviation of the average of the normal controls, and conversely, as hypomethylated if methylation values were below two times the standard deviation of the average of the normal control tissues. Differences in the degree of methylation were determined by two-sided non-parametric Mann-Whitney test for two-categorical clinicopathological factors and Kruskal-Wallis test for three-categorical clinicopathological factors. The $P$-values were obtained by permutation procedure without any assumption on specific distribution. Correlations between concomitant methylated genes were calculated using Spearman's test. All tests were performed using the Statistical Package for Science version 15.0. For all obtained p-values, the false discovery rate (FDR) was assessed using Benjamini and Hochberg [31] in the Bioconductor multitest package [32].

\section{Results}

\section{CpG promoter methylation patterns across three breast} cancer diagnosis groups

The results of the pyrosequencing analysis of 27 DCIS, 28 invasive tumours and 34 mixed tumours, are illustrated in Figure $1 \mathrm{~A}$ and $1 \mathrm{~B}$. All patient samples in all three groups displayed widespread aberrant $\mathrm{CpG}$ island methylation in the analyzed gene set. Aberrant DNA methylation (defined as an increase of average methylation levels beyond the average \pm two times the standard deviation observed in the group of normal tissues) was observed in all three diagnosis groups for the following genes (frequency; \% of DCIS vs. invasive vs. mixed): $A B C B 1(40.7 \%$ vs. $39.3 \%$ vs. $44.1 \%)$, FOXC1 (22.2\% vs. $53.6 \%$ vs. $67.6 \%)$, GSTP1 (22.2\% vs. $14.3 \%$ vs. $26.5 \%)$, MGMT (3.7\% vs. $3.6 \%$ vs. $5.9 \%), M L H 1$ (7.4\% vs. $3.6 \%$ vs. $2.9 \%), P P P 2 R 2 B(55.0 \%$ vs. $78.6 \%$ vs. $70.6 \%), C D K N 2 A / p 16^{I N K 4 a}(0 \%$ vs. $10.7 \%$ vs. $5.9 \%)$, PTEN (18.5\% vs. $14.3 \%$ vs. $23.5 \%)$ and $R A S S F 1 A$ (85.2\% vs. $82.1 \%$ vs. $85.3 \%)$. Methylation of $A B C B 1$, FOXC1, PTEN and PPP2R2B in DCIS is reported here for the first time (Figure 2). To rule out any artefact due to inter-individual variation in $A B C B 1, F O X C 1, P T E N$ and $P P P 2 R 2 B$ methylation patterns in normal tissue and to confirm the presence of DNA methylation to be a tumour specific event, we analyzed the methylation patterns in 23 additional normal tissues without histopathological findings, confirming the initial findings of an absence of DNA methylation around the transcription start site of these genes in normal breast tissue (Figures 2 and 3A). Overall, 


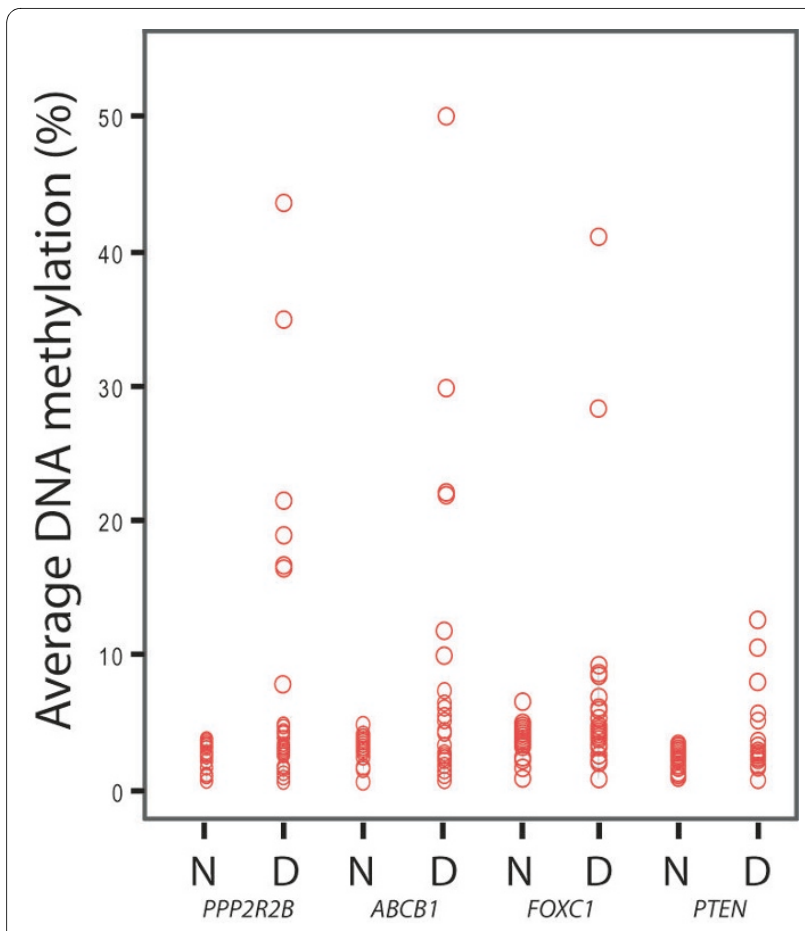

Figure $\mathbf{2}$ Newly identified aberrantly methylated genes in DCIS. Differences in the average DNA methylation (\%) between normal and DCIS tissue for the newly identified methylated genes in DCIS; PPP2R2B, $A B C B 1, F O X C 1$ and PTEN. The average DNA methylation (\%) value is the average value of methylation for all CpGs calculated for each sample. Abbreviations: $\mathrm{N}=$ normal tissue, $\mathrm{D}=\mathrm{DClS}$.

methylation was observed already in DCIS and the frequency (that is, the number of methylated samples) was unchanged with the advancement of the disease for most of the genes except for FOXC1. The frequency of FOXC1 methylated samples increased from in situ to invasive cancer. In addition, differential levels of DNA methylation (average DNA methylation) of FOXC1 among the diagnosis groups were observed. For FOXC1 significant differences in methylation levels were observed between normal breast tissue and IDC $(P<0.001)$ and mixed tumours $(P<0.001)$ (Figure 3A). Significantly lower levels of FOXC1 methylation were observed in DCIS compared to invasive and mixed tumours $(P=0.007$, and $P=0.001$, respectively). No significant difference in DNA methylation levels between the diagnosis groups was observed for any of the other genes studied. The number of methylated genes was not different between DCIS, invasive and mixed patients. ESR1 did not show any difference in methylation levels between tumours and normal tissues. The imprinted gene IGF2 was hypomethylated in 10 DCIS, 11 invasive and 5 mixed tumours and hypermethylated in 1 DCIS, 3 invasive and 10 mixed tumours.

Concomitant DNA methylation was observed between genes at different chromosomes. Methylation at FOXC1 (chr 6p) showed significant correlations to RASSF1A (chr 3p), PPP2R2B (chr 5q), ABCB1 (chr 7q) and GSTP1 (chr 11q) $\left(P=7.6^{*} 10^{-4}\right.$ and $\mathrm{R}^{2}=0.34, P=3.6^{*} 10^{-5}$ and $\mathrm{R}^{2}=0.41$, $P=4.5^{*} 10^{-6}$ and $\mathrm{R}^{2}=0.46, P=1.5^{*} 10^{-4}$ and $\mathrm{R}^{2}=0.38$, respectively). In addition methylation at PTEN (chr 10q) was significantly correlated to $A B C B 1$ (chr 7q) and $P P P 2 R 2 B$ (chr 5q) methylation $\left(P=1.4^{*} 10^{-6}, \mathrm{R}^{2}=0.48\right.$ and $P=1.0^{*} 10^{-6}, \mathrm{R}^{2}=0.60$, respectively). All $P$-values were obtained with a false discovery rate $<5 \%$.

\section{qRT-PCR validation of the expression of FOXC1}

The functional impact of FOXC1 methylation on its expression level was investigated by TaqMan qRT-PCR. Both methylated $(\mathrm{n}=19)$ and unmethylated $(\mathrm{n}=29)$ tumours showed significantly lower expression values compared to normal tissue ( $\mathrm{n}=28$, both $P<0.001$ ) (Figure $3 \mathrm{~B}$ ), indicating that the transcriptional inactivation of $F O X C 1$ is an early event during tumour progression.

\section{Methylation profiles associated with clinicopathological features}

Using the nonparametric Mann-Whitney test we found that FOXC1, GSTP1, ABCB1 and RASSF1A displayed a significantly different level of methylation between ER positive and ER negative samples $(P=0.009, P=0.003, P$ $=0.003$ and $P=0.003$, respectively). Specifically samples with a negative ER status showed the lowest degree of methylation (Figure 4A). FOXC1, ABCB1, PPP $2 R 2 B$ and $P T E N$ displayed significant differences in methylation levels in TP53 wild type and TP53 mutated samples $(P=$ $0.006, P=0.015, P=0.025$ and $P=0.01$, respectively) with the TP53 mutated samples having the lower DNA methylation (Figure 4B). In addition $A B C B 1$ methylation was lower in Ki67 positive tumours $(P=0.006)$ and GSTP1 methylation was lower in PR negative tumours $(P=$ 0.009) (Figure 4C). No statistically significant difference could be attributed to histopathological grade.

\section{Discussion}

In the present study we quantitatively determined the methylation levels in the promoter regions of 11 cancerrelated genes in DCIS, small invasive breast cancers, mixed lesions and normal breast tissues. Aberrant DNA methylation was already present in DCIS for several of the genes studied. No DNA methylation changes specific for invasive breast cancer were identified.

Previous candidate gene studies have investigated promoter hypermethylation of in situ lesions and have shown methylation for GSTP1, CyclinD2, RARB2, Twist,

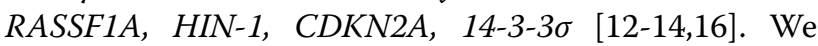
found frequencies of methylation for RASSF1A in both DCIS and invasive tumours similar to previously published reports ranging from 60 to $88 \%$ in different populations $[14,15]$. Results seem thus to be very consistent over 


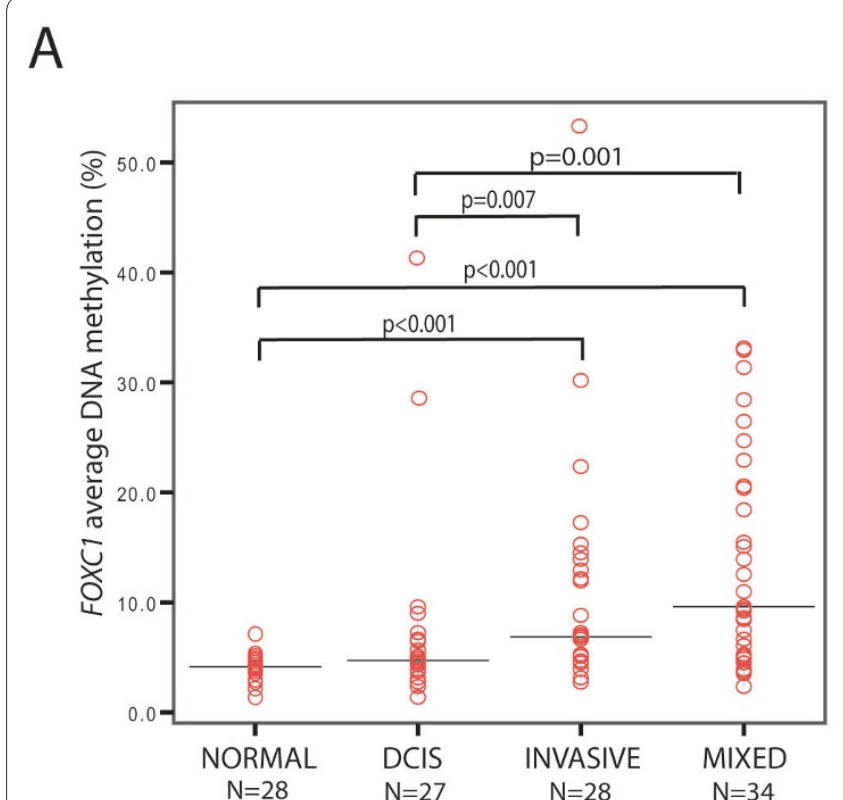

B

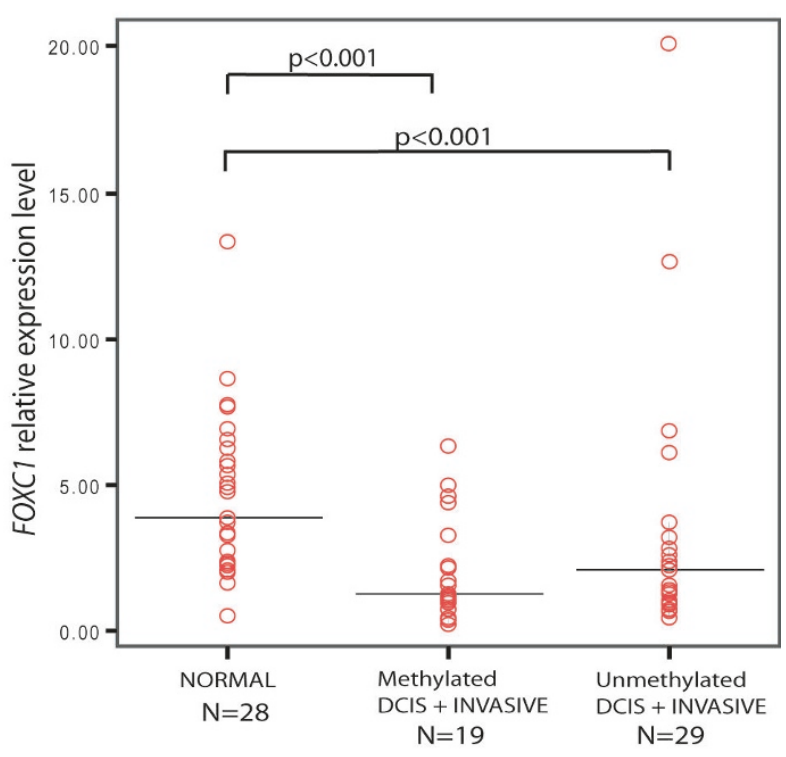

Figure 3 Differential FOXC1 methylation across diagnosis groups and subsequent validation by qRT-PCR. A: Differences in FOXC1 average DNA methylation (\%) between normal breast tissue and the different diagnosis groups. The FOXC1 average DNA methylation (\%) value is the average value of methylation for all CpGs calculated for each tumour sample. B: Differences in relative expression levels of FOXC1 as measured by qRT-PCR in normal breast tissue versus methylated and unmethylated tumours. Expression of FOXC1 was measured relative to the expression of the reference gene PGK1. Black horizontal bars represent median value for each diagnosis group.

different technology platforms identifying the epigenetic inactivation of RASSF1A as a very early step during breast carcinogenesis. GSTP1 has been found to be frequently methylated in different stages of breast carcinomas. Again, similar frequencies of 20 to $30 \%$ were found here for GSTP1 compared with recent reports [21,33-35]. In this study, methylation of MGMT was rare in both DCIS and invasive tumours, also in concordance with previous studies [36]. Infrequent methylation was also observed for the $M L H 1$ gene, in line with the absence of changes in $M L H 1$ expression during early breast carcinogenesis as assessed by IHC $[37,38]$. We also found minimal methylation of CDKN2A within the CpGs studied here, which is in concordance with a previous study on DCIS and other proliferative lesions of the breast [16]. No methylation of ESR1 was observed in this study, although we have used the commonly studied region $400 \mathrm{bp}$ downstream of ESR1 transcription start site. A previous study observed methylation in DCIS samples [15], however they could not show significant differences between normal and diseased tissue in North-American and Korean populations. A study by Feng et al. (2007) shows the same result as we report with no increase in ESR1 methylation in malignant compared to normal breast tissues [39]. However, we can not exclude the possibility that the reason why we are not able to detect any ESR1 methylation is that pyrosequenc- ing is less sensitive compared to the Q-MSP technology used in [15].

A novel finding of our study was the identification of aberrant DNA methylation of $A B C B 1, F O X C 1, P P P 2 R 2 B$ and PTEN in DCIS. The methylation frequencies were similar in all diagnosis groups for $A B C B 1, P P P 2 R 2 B$ and PTEN. PTEN and PPP $2 R 2 B$ are both candidate tumour suppressor genes $[23,40]$ and our results suggest that epigenetic silencing might be involved in dysregulation of these genes in DCIS. We have observed the methylation of $P P P 2 R 2 B$ in locally advanced breast tumours (Dejeux et al, submitted for publication), and PTEN has been found to be frequently methylated in breast carcinomas [24,41]. $A B C B 1$ is an ATP dependent p-glycoprotein involved in the efflux of various small molecules and xenobiotics in extra and intracellular membranes. Association of DNA methylation of $A B C B 1$ and drug resistance in breast cancer cell-lines has been reported [42], and $A B C B 1$ expression has been associated with poor outcome in breast cancer patients [43]. We have observed that $A B C B 1$ methylation is important for treatment response and overall survival in patients with advanced breast cancer treated with doxorubicin (Dejeux E, et al. submitted). In this study, $A B C B 1$ methylation was associated with non-proliferative, Ki67 negative tumours supporting a positive role for $A B C B 1$ methylation in breast 


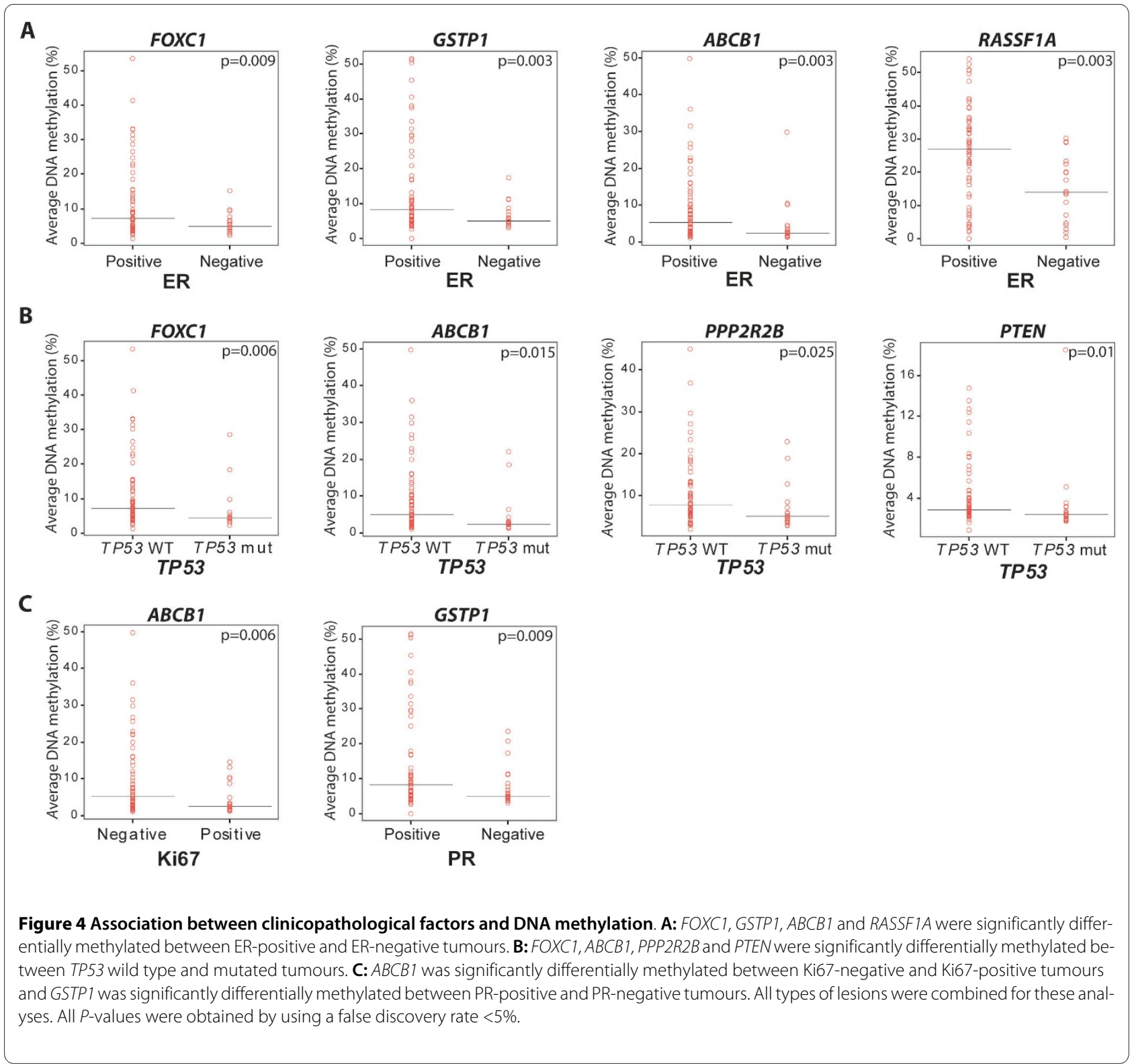

cancer progression and outcome. FOXC1 is a transcription factor with an important role in the regulation of ocular development [44]. FOXC1 is hypomethylated and highly expressed in CD44+ breast progenitor cells and might play an important role in the differentiation of mammary epithelial cell phenotypes [45]. In our data set, FOXC1 displayed a significantly increased methylation levels from normal breast tissue to invasive tumours with simultaneously lower FOXC1 gene expression as measured by qRT-PCR. The tumours with less methylation and somewhat higher expression of FOXC1 (Figure 3B) tended to be of the basal-like and normal-like breast cancer subtypes, as determined by gene expression profiling. Luminal B-like and ERBB2-like tumours had significantly lower FOXC1 expression $(P=0.026$ and $P=0.018$, respectively) compared to the basal-like tumours whereas no statistical significance was found for basal vs. luminal A-like tumours $(P=0.134)$ (Muggerud A et al., unpublished results). This supports the view of heterogeneous FOXC1 expression across molecular subtypes, which is in concordance with previously reported results [45]. Bloushtain-Qimron et al reported also increased methylation in matched distant metastases compared to the primary tumours, supporting a differential role for FOXC1methylated cells in the progression of the disease. The 28 normal breast tissue samples analysed in this study displayed on average significantly higher levels of FOXC1 gene expression compared to the DCIS, small invasive and mixed lesions. Both the methylated and unmethylated DCISs and invasive tumours displayed significantly 
lower levels of FOXC1 gene expression. This might indicate that histone modifications or other mechanisms in addition to promoter hypermethylation silence FOXC1 in the unmethylated tumours. Further studies are needed to investigate the functional consequence of this increase of DNA methylation and the potential role of FOXC1 in the progression from DCIS to invasive carcinoma.

Concomitant DNA methylation was observed between some of the genes studied suggesting connected epigenetic programs within tumours. In line with our result, significant correlation between GSTP1 and RASSF1A hypermethylation has previously been reported [46]. The chromosomal region 6p25 harbouring the FOXC1 gene is frequently gained in ER negative tumours [47] in line with our observation of low DNA methylation and high expression in basal and normal-like tumours. The other chromosomal regions harbouring the genes with a high correlation to FOXC1 methylation have all been reported to either have gain or losses in breast cancer [48-50]. It is possible that concomitant DNA hypomethylation in these regions could induce chromosomal instability of the same regions as reported in colon cancer [51].

By unsupervised hierarchical clustering analysis of a number of methylation markers and tumours from 148 breast cancer patients, Widschwendter et al. (2004) [52], showed that the tumours segregated naturally into groups with distinct methylation profiles that differed significantly in their hormone receptor status. Further, other studies have focused on the epigenetic differences between ER positive and ER negative breast cancers and their results imply that methylation profiles of ER-positive tumours are different from those of ER-negative tumours [39,46]. Moreover they found that promoter hypermethylation of RASSF1A and GSTP1 was more frequent in ER-positive than in ER-negative tumours in both early and advanced breast tumours. Our data are consistent with these previous reports suggesting that ER (or hormone receptor) expression may influence epigenetic changes.

Lower levels of DNA methylation were observed in TP53 mutated tumours, especially at FOXC1, ABCB1, $P P P 2 R 2 B$ and PTEN promoters. The association of $A B C B 1$ and $P P P 2 R 2 B$ with TP53 status is also observed in more advanced tumours (Dejeux et al. submitted). It has also been reported that breast tumours with TP53 mutations lacked methylation in a number of regulatory genes [39]. Further in concordance with our results, Toyota et al. [53] found a high number of TP53 mutations in unmethylated colorectal tumours suggesting that TP53 mutations and epigenetic alterations of other growthsuppressing genes can be two distinct mechanisms that inactivate tumour-suppressor genes in breast cancer. Similar to the results of [52] we could not find associations between histopathological grade and DNA methyla- tion patterns among the samples and genes investigated. This might be due to independency of grade for this limited gene panel investigated or the relatively small cohort size.

Almost all genes methylated in DCIS and IDC have been identified using candidate gene approaches. Genome-wide methylation studies might provide useful in identifying new DNA methylation events occurring during early breast tumourigenesis. Since this study was designed to find differences related to tumour progression from in situ to invasive breast cancer, follow-up studies are needed to investigate these biological markers and their potential in predicting prognosis.

\section{Conclusions}

This study has identified four novel genes as methylated in DCIS; ABCB1, FOXC1, PPP2R2B and PTEN. Their role in the progression from in situ to invasive carcinoma needs further investigation. Furthermore, these analyses demonstrate that promoter methylation is an early and frequent event in breast cancer and most of the genes that are found to be methylated in advanced breast tumours are already found methylated in DCIS.

\section{Additional material}

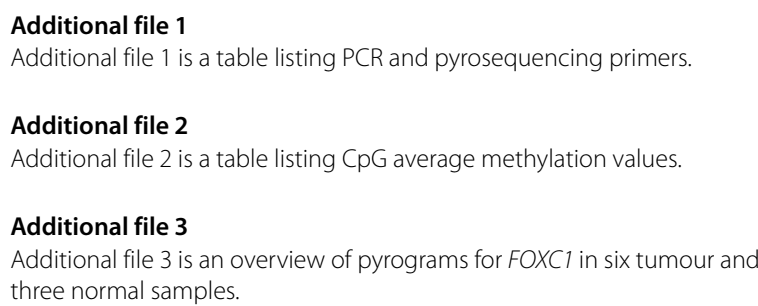

\section{Abbreviations}

DCIS: ductal carcinoma in situ; ER: estrogen receptor; FDR: false discovery rate; IDC: invasive ductal carcinoma; IHC: immunohistochemistry; PR: progesterone receptor; PCR: polymerase chain reaction; q-MSP: quantitative methyl-specific PCR; qRT-PCR: quantitative real-time PCR.

\section{Competing interests}

The authors declare that they have no competing interests.

\section{Authors' contributions}

AAM performed laboratory experiments, data analyses and wrote the manuscript. JAR performed laboratory experiments, data analyses and wrote the manuscript. FW was responsible for the patient cohorts, involved in the study design and wrote the manuscript. JB was responsible for the fresh frozen sample cohorts. FB and $J$ J were involved in laboratory experiments. HS was involved in the statistical analyses. IB provided normal breast tissue samples. ALBD, VNK, TS and JT initiated and designed the study and were involved in writing the manuscript. All authors have read and approved the final manuscript.

\section{Acknowledgements}

This study was supported by the Norwegian Cancer Society (PR-2006-0332 to TS and D-03067 to VNK), the Norwegian Research Council (163027/N409 to TS), 
the French-Norwegian cooperation program Aurora grant 15842WE (to VNK and JT) and the Swedish Cancer Society (4920-B06-03PCD to FW). AAM is a fellow of the Norwegian Cancer Society. JAR is a fellow of the Faculty of Medicine, University of Oslo.

\section{Author Details}

'Department of Genetics, Institute for Cancer Research, Oslo University Hospital Radiumhospitalet, Montebello, Oslo, N-0310, Norway,

${ }^{2}$ Faculty of Medicine, Division The Norwegian Radium Hospital, University of Oslo, Montebello, Oslo, N-0310, Norway,

${ }^{3}$ Department of Surgery, Uppsala University Hospital, Dag Hammarskjölds väg 20, Uppsala, SE-751 85, Sweden,

4Department of Genetics and Pathology, Uppsala University Hospital, Dag Hammarskjölds väg 20, Uppsala, SE-751 85, Sweden,

5Institute for Clinical Epidemiology and Molecular Biology (EpiGen), Faculty of Medicine, Division Akershus University Hospital, University of Oslo,

Sykehusveien 27, Nordbyhagen, N-1474, Norway,

6Department of Surgery, Akershus University Hospital, Sykehusveien 27,

Nordbyhagen, N-1474, Norway,

7Faculty of Medicine, Division Akershus University Hospital, Sykehusveien 27, Nordbyhagen, N-1474, Norway,

${ }^{8}$ Biomedical Research Group, Department of Informatics, University of Oslo, P.O. Box 1080 Blindern, Oslo, N-0316, Norway,

'Laboratory for Epigenetics, Centre National de Génotypage, CEA-Institut de Génomique, 2 rue Gaston Crèmieux, 91000 Evry, France and

${ }^{10}$ Department of Biostatistics, Institute of Basic Medical Science, University of Oslo, P.O. Box 1122 Blindern, Oslo, N-0317, Norway

Received: 26 March 2009 Revisions Requested: 15 May 2009 Revised: 2 December 2009 Accepted: 7 January 2010 Published: January 2010

\section{References}

1. Chin K, de Solorzano CO, Knowles D, Jones A, Chou W, Rodriguez EG, Kuo WL, Ljung BM, Chew K, Myambo K, Miranda M, Krig S, Garbe J, Stampfer M, Yaswen P, Gray JW, Lockett SJ: In situ analyses of genome instability in breast cancer. Nat Genet 2004, 36:984-988.

2. Wiechmann $L$, Kuerer $H M$ : The molecular journey from ductal carcinoma in situ to invasive breast cancer. Cancer 2008, 112:2130-2142

3. Yao J, Weremowicz S, Feng B, Gentleman RC, Marks JR, Gelman R, Brennan C, Polyak K: Combined cDNA array comparative genomic hybridization and serial analysis of gene expression analysis of breast tumor progression. Cancer Res 2006, 66:4065-4078.

4. Ma XJ, Salunga R, Tuggle JT, Gaudet J, Enright E, McQuary P, Payette T, Pistone M, Stecker K, Zhang BM, Zhou YX, Varnholt H, Smith B, Gadd M, Chatfield E, Kessler J, Baer TM, Erlander MG, Sgroi DC: Gene expression profiles of human breast cancer progression. Proc Natl Acad Sci USA 2003, 100:5974-5979.

5. Schuetz CS, Bonin M, Clare SE, Nieselt K, Sotlar K, Walter M, Fehm T, Solomayer E, Riess O, Wallwiener D, Kurek R, Neubauer HJ: Progressionspecific genes identified by expression profiling of matched ductal carcinomas in situ and invasive breast tumors, combining laser capture microdissection and oligonucleotide microarray analysis. Cancer Res 2006, 66:5278-5286

6. Porter D, Lahti-Domenici J, Keshaviah A, Bae YK, Argani P, Marks J, Richardson A, Cooper A, Strausberg R, Riggins GJ, Schnitt S, Gabrielson E, Gelman R, Polyak K: Molecular markers in ductal carcinoma in situ of the breast. Mol Cancer Res 2003, 1:362-375.

7. Hannemann J, Velds A, Halfwerk JB, Kreike B, Peterse JL, Vijver MJ van de: Classification of ductal carcinoma in situ by gene expression profiling. Breast Cancer Res 2006, 8:R61.

8. Lukas J, Niu N, Press MF: p53 mutations and expression in breast carcinoma in situ. Am J Pathol 2000, 156:183-191.

9. Done SJ, Eskandarian S, Bull S, Redston M, Andrulis IL: p53 missense mutations in microdissected high-grade ductal carcinoma in situ of the breast. J Natl Cancer Inst 2001, 93:700-704.

10. Zhou W, Muggerud AA, Vu P, Due EU, Sorlie T, Borresen-Dale AL, Warnberg F, Langerod A: Full sequencing of TP53 identifies identical mutations within in situ and invasive components in breast cancer suggesting clonal evolution. Mol Oncol 2009 in press.
11. Jones PA, Baylin SB: The epigenomics of cancer. Cell 2007, 128:683-692.

12. Lee JS: GSTP1 promoter hypermethylation is an early event in breast carcinogenesis. Virchows Arch 2007, 450:637-642.

13. Evron E, Dooley WC, Umbricht CB, Rosenthal D, Sacchi N, Gabrielson E, Soito AB, Hung DT, Ljung B, Davidson NE, Sukumar S: Detection of breast cancer cells in ductal lavage fluid by methylation-specific PCR. Lancet 2001, 357:1335-1336.

14. Fackler MJ, McVeigh M, Evron E, Garrett E, Mehrotra J, Polyak K, Sukumar S, Argani P: DNA methylation of RASSF1A, HIN-1, RAR-beta, Cyclin D2 and Twist in in situ and invasive lobular breast carcinoma. Int J Cancer 2003, 107:970-975

15. Lee JS, Fackler MJ, Teo WW, Lee JH, Choi C, Park MH, Yoon JH, Zhang Z, Argani P, Sukumar S: Quantitative promoter hypermethylation profiles of ductal carcinoma in situ in North American and Korean women: Potential applications for diagnosis. Cancer Biol Ther 2008, 7:1398-1406.

16. Lehmann U, Langer F, Feist $\mathrm{H}$, Glockner S, Hasemeier B, Kreipe H: Quantitative assessment of promoter hypermethylation during breast cancer development. Am J Pathol 2002, 160:605-612.

17. Honorio S, Agathanggelou A, Schuermann M, Pankow W, Viacava P, Maher ER, Latif F: Detection of RASSF1A aberrant promoter hypermethylation in sputum from chronic smokers and ductal carcinoma in situ from breast cancer patients. Oncogene 2003, 22:147-150.

18. David GL, Yegnasubramanian S, Kumar A, Marchi VL, De Marzo AM, Lin X Nelson WG: MDR1 promoter hypermethylation in MCF-7 human breast cancer cells: changes in chromatin structure induced by treatment with 5-Aza-cytidine. Cancer Biol Ther 2004, 3:540-548.

19. Esteller M, Corn PG, Baylin SB, Herman JG: A gene hypermethylation profile of human cancer. Cancer Res 2001, 61:3225-3229.

20. Lapidus RG, Nass SJ, Butash KA, Parl FF, Weitzman SA, Graff JG, Herman JG, Davidson NE: Mapping of ER gene CpG island methylation-specific polymerase chain reaction. Cancer Res 1998, 58:2515-2519.

21. Esteller M, Corn PG, Urena JM, Gabrielson E, Baylin SB, Herman JG: Inactivation of glutathione S-transferase P1 gene by promoter hypermethylation in human neoplasia. Cancer Res 1998, 58:4515-4518.

22. Issa JP, Vertino PM, Boehm CD, Newsham IF, Baylin SB: Switch from monoallelic to biallelic human IGF2 promoter methylation during aging and carcinogenesis. Proc Natl Acad Sci USA 1996, 93:11757-11762.

23. Keen JC, Garrett-Mayer E, Pettit C, Mack KM, Manning J, Herman JG, Davidson NE: Epigenetic regulation of protein phosphatase 2A (PP2A), lymphotactin (XCL1) and estrogen receptor alpha (ER) expression in human breast cancer cells. Cancer Biol Ther 2004, 3:1304-1312.

24. Garcia JM, Silva J, Pena C, Garcia V, Rodriguez R, Cruz MA, Cantos B, Provencio M, Espana P, Bonilla F: Promoter methylation of the PTEN gene is a common molecular change in breast cancer. Genes Chromosomes Cancer 2004, 41:117-124.

25. Yan PS, Shi H, Rahmatpanah F, Hsiau TH, Hsiau AH, Leu YW, Liu JC, Huang TH: Differential distribution of DNA methylation within the RASSF1A CpG island in breast cancer. Cancer Res 2003, 63:6178-6186.

26. Holland R, Peterse JL, Millis RR, Eusebi V, Faverly D, Vijver M van de, Zafrani B: Ductal carcinoma in situ: a proposal for a new classification. Semin Diagn Pathol 1994, 11:167-180

27. Elston CW, Ellis IO: Pathological prognostic factors in breast cancer. I. The value of histological grade in breast cancer: experience from a large study with long-term follow-up. Histopathology 1991, 19:403-410.

28. Tost J, Gut IG: DNA methylation analysis by pyrosequencing. Nat Protoc 2007, 2:2265-2275.

29. Kuijk EW, du Puy L, van Tol HT, Haagsman HP, Colenbrander B, Roelen BA: Validation of reference genes for quantitative RT-PCR studies in porcine oocytes and preimplantation embryos. BMC Dev Biol 2007, 7:58

30. Boda E, Pini A, Hoxha E, Parolisi R, Tempia F: Selection of reference genes for quantitative real-time RT-PCR studies in mouse brain. $J$ Mol Neurosci 2009, 37:238-253.

31. Benjamini $Y$, Hochberg Y: Controlling the false discovery rate: a practical and powerful approach to multiple testing. J Royal Stat $\operatorname{Soc} \operatorname{Ser} B$ 1995:289-300

32. Bioconductor [http://www.bioconductor.org/

33. Ronneberg JA, Tost J, Solvang HK, Alnaes GI, Johansen FE, Brendeford EM, Yakhini Z, Gut IG, Lonning PE, Borresen-Dale AL, Gabrielsen OS, Kristensen VN: GSTP1 promoter haplotypes affect DNA methylation levels and promoter activity in breast carcinomas. Cancer Res 2008, 68:5562-5571. 
34. Pasquali L, Bedeir A, Ringquist S, Styche A, Bhargava R, Trucco G: Quantification of $\mathrm{CpG}$ island methylation in progressive breast lesions from normal to invasive carcinoma. Cancer Lett 2007, 257:136-144.

35. Parrella P, Poeta ML, Gallo AP, Prencipe M, Scintu M, Apicella A, Rossiello R, Liguoro G, Seripa D, Gravina C, Rabitti C, Rinaldi M, Nicol T, Tommasi S, Paradiso A, Schittulli F, Altomare V, Fazio VM: Nonrandom distribution of aberrant promoter methylation of cancer-related genes in sporadic breast tumors. Clin Cancer Res 2004, 10:5349-5354

36. Viswanathan M, Solomon SP, Tsuchida N, Selvam GS, Shanmugam G: Methylation of E-cadherin and hMLH1 genes in Indian sporadic breast carcinomas. Indian J Exp Biol 2006, 44:115-119.

37. Batistatou A, Arkoumani E, Stefanou D: Expression of mismatch repair proteins in invasive and in situ carcinoma of the breast. In Vivo 2004, 18:661-666.

38. Khilko N, Bourne P, Qi Y, Ping T: Mismatch repair genes hMLH1 and $\mathrm{hMSH} 2$ may not play an essential role in breast carcinogenesis. Int J Surg Pathol 2007, 15:233-241.

39. Feng W, Shen L, Wen S, Rosen DG, Jelinek J, Hu X, Huan S, Huang M, Liu J, Sahin AA, Hunt KK, Bast RC Jr, Shen Y, Issa JP, Yu Y: Correlation between CpG methylation profiles and hormone receptor status in breast cancers. Breast Cancer Res 2007, 9:R57.

40. Simpson L, Parsons R: PTEN: life as a tumor suppressor. Exp Cell Res 2001, 264:29-41.

41. Khan S, Kumagai T, Vora J, Bose N, Sehgal I, Koeffler PH, Bose S: PTEN promoter is methylated in a proportion of invasive breast cancers. Int $J$ Cancer 2004, 112:407-410.

42. Reed K, Hembruff SL, Laberge ML, Villeneuve DJ, Cote GB, Parissenti AM: Hypermethylation of the $A B C B 1$ downstream gene promoter accompanies $A B C B 1$ gene amplification and increased expression in docetaxel-resistant MCF-7 breast tumor cells. Epigenetics 2008, 3:270-280.

43. Atalay C, Demirkazik A, Gunduz U: Role of $A B C B 1$ and $A B C C 1$ gene induction on survival in locally advanced breast cancer. $J$ Chemother 2008, 20:734-739.

44. Lehmann OJ, Sowden JC, Carlsson P, Jordan T, Bhattacharya SS: Fox's in development and disease. Trends Genet 2003, 19:339-344.

45. Bloushtain-Qimron N, Yao J, Snyder EL, Shipitsin M, Campbell LL, Mani SA, Hu M, Chen H, Ustyansky V, Antosiewicz JE, Argani P, Halushka MK, Thomson JA, Pharoah P, Porgador A, Sukumar S, Parsons R, Richardson AL, Stampfer MR, Gelman RS, Nikolskaya T, Nikolsky Y, Polyak K: Cell typespecific DNA methylation patterns in the human breast. Proc Natl Acad Sci USA 2008, 105:14076-14081.

46. Sunami E, Shinozaki M, Sim MS, Nguyen SL, Vu AT, Giuliano AE, Hoon DS: Estrogen receptor and HER2/neu status affect epigenetic differences of tumor-related genes in primary breast tumors. Breast Cancer Res 2008, 10:R46.

47. Bergamaschi A, Kim YH, Wang P, Sorlie T, Hernandez-Boussard T, Lonning PE, Tibshirani R, Borresen-Dale AL, Pollack JR: Distinct patterns of DNA copy number alteration are associated with different clinicopathological features and gene-expression subtypes of breast cancer. Genes Chromosomes Cancer 2006, 45:1033-1040.

48. Chin K, DeVries S, Fridlyand J, Spellman PT, Roydasgupta R, Kuo WL, Lapuk A, Neve RM, Qian Z, Ryder T, Chen F, Feiler H, Tokuyasu T, Kingsley C, Dairkee S, Meng Z, Chew K, Pinkel D, Jain A, Ljung BM, Esserman L, Albertson DG, Waldman FM, Gray JW: Genomic and transcriptional aberrations linked to breast cancer pathophysiologies. Cancer Cell 2006, 10:529-541.

49. Adelaide J, Finetti P, Bekhouche I, Repellini L, Geneix J, Sircoulomb F, Charafe-Jauffret E, Cervera N, Desplans J, Parzy D, Schoenmakers E, Viens P, Jacquemier J, Birnbaum D, Bertucci F, Chaffanet M: Integrated profiling of basal and luminal breast cancers. Cancer Res 2007, 67:11565-11575.

50. Pinto $A E$, Roque $L$, Rodrigues $R$, Andre $S$, Soares J: Frequent 7q gains in flow cytometric multiploid/hypertetraploid breast carcinomas: a study of chromosome imbalances by comparative genomic hybridisation. $J$ Clin Pathol 2006, 59:367-372.

51. Rodriguez J, Frigola J, Vendrell E, Risques RA, Fraga MF, Morales C, Moreno V, Esteller M, Capella G, Ribas M, Peinado MA: Chromosomal instability correlates with genome-wide DNA demethylation in human primary colorectal cancers. Cancer Res 2006, 66:8462-9468.
52. Widschwendter M, Siegmund KD, Muller HM, Fiegl H, Marth C, MullerHolzner E, Jones PA, Laird PW: Association of breast cancer DNA methylation profiles with hormone receptor status and response to tamoxifen. Cancer Res 2004, 64:3807-3813.

53. Toyota M, Ohe-Toyota M, Ahuja N, Issa JP: Distinct genetic profiles in colorectal tumors with or without the $\mathrm{CpG}$ island methylator phenotype. Proc Natl Acad Sci USA 2000, 97:710-715.

\section{doi: $10.1186 / \mathrm{bcr} 2466$}

Cite this article as: Muggerud et al., Frequent aberrant DNA methylation of $A B C B 1, F O X C 1$, PPP2R2B and PTEN in ductal carcinoma in situ and early invasive breast cancer Breast Cancer Research 2010, 12:R3

\section{Submit your next manuscript to BioMed Central and take full advantage of:}

- Convenient online submission

- Thorough peer review

- No space constraints or color figure charges

- Immediate publication on acceptance

- Inclusion in PubMed, CAS, Scopus and Google Scholar

- Research which is freely available for redistribution
C Biomed Central 